\title{
Erratum to: Systematic review and meta-analysis of randomized controlled trials evaluating silodosin in the treatment of non-neurogenic male lower urinary tract symptoms suggestive of benign prostatic enlargement
}

\author{
Giacomo Novara $\cdot$ Andrea Tubaro $\cdot$ Roberto Sanseverino $\cdot$ \\ Sebastiano Spatafora $\cdot$ Walter Artibani $\cdot$ Filiberto Zattoni • \\ Francesco Montorsi · Christopher R. Chapple
}

Published online: 4 July 2013

(c) Springer-Verlag Berlin Heidelberg 2013

\section{Erratum to: World J Urol}

DOI 10.1007/s00345-012-0944-8

The author would like to correct the errors in the publication of the original article. The corrected details are given below for your reading.

Firstly, on page 1, in the abstract paragraph under the heading results, "and $\mathrm{Q}_{\max }(p \leq 0.05)$ )" has to be removed and the revised text should read as

"Silodosin was more effective than tamsulosin $0.2 \mathrm{mg}$ with regard to some IPSS-related parameters."

Secondly, on page 6, 2nd paragraph under the heading "Silodosin versus tamsulosin," the text is amended with few changes and the revised text should read as

"Silodosin was more effective than tamsulosin $0.2 \mathrm{mg}$ with regard to mean change in IPSS voiding subscore (WMD: $-0.75 ; p=0.03$ ) (Fig. 2c), and IPSS quality-of-

The online version of the original article can be found under doi:10.1007/s00345-012-0944-8.

G. Novara $(\bowtie) \cdot$ F. Zattoni

Department of Oncological, Surgical, and Gastroenterological

Sciences, Urology Clinic, University of Padua,

Via Giustiniani 2, 35100 Padua, Italy

e-mail: giacomonovara@gmail.com; giacomo.novara@unipd.it

A. Tubaro

Department of Urology, Sant'Andrea Hospital,

La Sapienza University, Rome, Italy

R. Sanseverino

Ospedale Umberto I, Salerno, Italy

S. Spatafora

Azienda Ospedaliera Santa Maria Nuova, Reggio Emilia, Italy life item (WMD: $-0.26 ; p=0.02$ ) (Fig. 2d). Conversely, only a non-statistically significant trend in favor of silodosin when compared with tamsulosin $0.2 \mathrm{mg}$ was found with regard to mean change in IPSS total score (WMD: $-1.48 ; p=0.08$ ) (Fig. 2a) and IPSS storage subscore (WMD: $-0.31 ; p=0.14$ ) (Fig. 2b). Silodosin and tamsulosin $0.4 \mathrm{mg}$ were at least as effective in all the efficacy analyses (Fig. 2a-e)."

Finally, on page 10, last sentence of the 1st paragraph should read as

"However, post hoc analyses of the European registrational trial found a significant difference in favor of silodosin in comparison with tamsulosin 0.4 for 3 IPSS symptoms (incomplete emptying, frequency, nocturia) considered simultaneously in patients with either moderate or severe LUTS [24]."

W. Artibani

University of Verona, Verona, Italy

F. Montorsi

Urologic Research Institute, Vita Salute San

Raffaele University, Milan, Italy

C. R. Chapple

Sheffield Teaching Hospitals NHS Foundation Trust,

Royal Hallamshire Hospital, Glossop Road,

Sheffield S10 2JF, UK 